\title{
Induction of anti-tumor immunity by lyophilized myeloma cells secreting GM-CSF
}

\author{
SHENG WU*, DAWEN YUAN*, JIAN-NING LIU and XIANG-YANG TAN \\ Institute of Molecular Medicine, Nanjing University, Nanjing 210093, P.R. China
}

Received August 1, 2006; Accepted November 2, 2006

\begin{abstract}
We have previously demonstrated that a low dose of live myeloma FO cells induced a cellular immunity against tumor without additional modulating factors. In the present study, lyophilized myeloma FO cells were used to induce anti-tumor immunity. In a myeloma vaccination model, immunization with lyophilized myeloma FO cells alone induced a slight response. However, the immunity was dramatically enhanced by myeloma FO cells transfected with a recombinant adenovirus, Adv-1/GM-CSF. The immunocytochemical staining of Adv-1/GM-CSF transfected myeloma FO cells confirmed that more than $90 \%$ of cells were positive with GM-CSF expression. Results of sandwich ELISA showed the amount of secreted GM-CSF was $240 \mathrm{ng} / 24 \mathrm{~h}$ per $10^{6}$ cells. Immunization with lyophilized myeloma FO cells secreting GM-CSF prevented the tumor growth in $60 \%$ of $\mathrm{BALB} / \mathrm{c}$ mice. Antibodies against myeloma FO cells were found in the sera of immunized mice. Tumor-specific T-cell response was also evaluated using cytotoxic T lymphocyte assay. In conclusion, lyophilized myeloma FO cells secreting GM-CSF can be used as a potent vaccine to induce strong and protective anti-tumor immunity.
\end{abstract}

\section{Introduction}

Based on the discovery of oncogenes (1), it has been accepted that tumor cells derive from normal tissues. Tumorigenesis comprises a number of cascade events, which decrease the immunogenicity of tumor cells to avoid the immune surveillance from their hosts (2). However, tumor-specific antigens or tumor-associated antigens also emerged during the course of tumorigenesis (3-6). They provided the potential targets for immunotherapy against cancer.

Correspondence to: Dr Jian-Ning Liu or Dr Xiang-Yang Tan, Institute of Molecular Medicine, Nanjing University, 22 Hankou Road, Nanjing 210093, P.R. China

E-mail: jianningliu2000@yahoo.com xiangyangtan@yahoo.com

${ }^{*}$ Contributed equally

Key words: GM-CSF, lyophilization, myeloma, tumor immunotherapy
Multiple strategies have been attempted to evoke anti-tumor immunity. Both humoral and cellular immunity demonstrated their therapeutic potentials $(7,8)$. A number of tumor antigens have been identified, such as MART-1, gp100 and TRP-2 $(3,5,6)$. Tumor cell surface antigens, such as CD20 on malignant B lymphomas and CD33 on leukemic blasts, were then used to induce tumor-specific antibodies, respectively (9). Her2-specific antibody, Herceptin, was a prominent example in the treatment of Her2-overexpressing metastatic breast cancer (10). Modified tumor cells, tumor peptide pulsed dendritic cells and tumor-dendritic fusion cells could also evoke immunity against cancer (11-13). Transfected tumor cells expressed costimulatory molecules, while dendritic cells presented tumor antigens by pulsing or cell fusion. Tumor-specific cytotoxic T lymphocytes (CTL) were usually activated in these strategies. Additionally, cytokines have demonstrated their helpful effects to anti-tumor immune responses. IL-2 and IL-12 have shown their therapeutic effects in clinical applications $(14,15)$, while reengineered tumor cells with GM-CSF increased the immunogenicity of irradiated tumor cells (16). In these methods, lymphocytes were recruited and activated by these cytokines. These studies suggested that anti-tumor immunity could be induced by different methods and play roles via different mechanisms.

We have demonstrated that a low dose of live myeloma FO cells induced protective anti-tumor immunity without any additional immune modulating factors (17). The method elicited a potent CTL response, which prevented tumorigenesis from challenge with $1 \times 10^{6}$ myeloma FO cells. In the present study, we investigated whether lyophilized GM-CSF secreting FO could also induce a protective anti-tumor immunity. Our data suggested that the immunity induced by lyophilized myeloma was dramatically enhanced by reengineering tumor cells to express GM-CSF. The mechanism of the anti-tumor immunity has been also studied.

\section{Materials and methods}

Mice and cell lines. Female BALB/c mice aged 6-8 weeks were purchased from the Shanghai Laboratory Animal Center. All procedures in animal experiments were approved by the Animal Study Committee, Institute of Molecular Medicine, Nanjing University. BALB/c myeloma cell line FO was obtained from ATCC. FO cells were maintained in RPMI1640 medium (Gibco, Grand Island, NY) supplemented with $10 \%$ heat-inactivated newborn calf serum (NCS) (Gibco), 

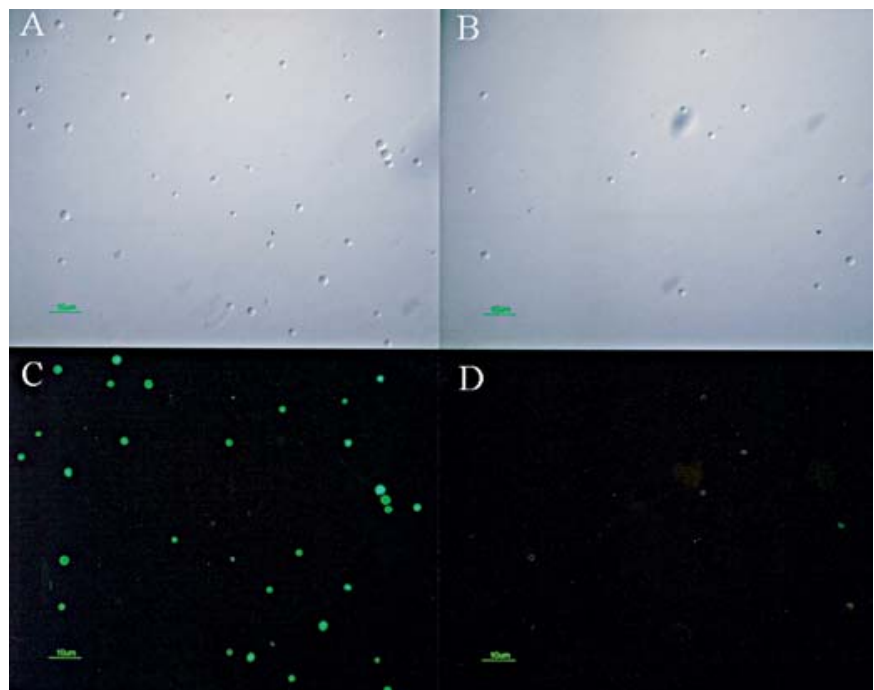

Figure 1. Immunocytochemical staining of myeloma FO cells transfected with Adv-1/GM-CSF. (A) Myeloma FO cells transfected with Adv-1/GMCSF (visual light); (B) myeloma FO cells without transfection (visual light); (C) myeloma FO cells transfected with Adv-1/GM-CSF (fluorescence) and (D) myeloma FO cells without transfection (fluorescence). Original magnification, $\mathrm{x} 200$.

2 mM L-glutamine (Hyclone, Logan, UT), $2 \mathrm{mg} / \mathrm{ml}$ sodium bicarbonate (Amersco, Cleveland, OH), 25 mM HEPES (Promega, Madison, WI), $100 \mathrm{U} / \mathrm{ml}$ penicillin (North China Pharmaceutical Group, Shijiazhuang, P.R. China) and $100 \mu \mathrm{g} /$ $\mathrm{ml}$ streptomycin (Lu-Kang Pharmaceuticals, Jining, P.R. China). Freshly isolated T-cells from mice spleen were cultured in RPMI-1640 medium supplemented with 10\% heat-inactivated fetal calf serum (FCS) (Gibco), $50 \mathrm{U} / \mathrm{ml}$ recombinant human interleukin 2 (IL-2) (Four Rings Biopharmaceuticals, Beijing, P.R. China), $5 \mu \mathrm{g} / \mathrm{ml}$ concanavalin A (Promega), $2 \mathrm{mM}$ L-glutamine, $2 \mathrm{mg} / \mathrm{ml}$ sodium bicarbonate, $25 \mathrm{mM}$ HEPES, $100 \mathrm{U} / \mathrm{ml}$ penicillin and $100 \mu \mathrm{g} / \mathrm{ml}$ streptomycin.

Transfection of myeloma FO cells with recombinant adenovirus $A d v-1 / G M-C S F$. FO cells were incubated with recombinant adenovirus containing GM-CSF (Adv-1/GM-CSF) (Institute of Molecular Medicine, Nanjing University, P.R. China), which was diluted 10 times by RPMI-1640 without serum, at $37^{\circ} \mathrm{C}$ for $1 \mathrm{~h}$. FO cells transfected with Adv-1/GM-CSF were maintained in RPMI-1640 supplemented with $10 \%$ NCS for 2 days.

Lyophilization of myeloma FO cells. Myeloma FO cells transfected with Adv-1/GM-CSF and normal myeloma FO cells were harvested and counted. Cells were washed with PBS 3 times and aliquoted to $1 \times 10^{6}$ per vials. After decanting the supernatant and freezing the cell pellets at $-80^{\circ} \mathrm{C}$ for $1 \mathrm{~h}$, cells were lyophilized by using a freeze-dryer (FTS systems, Stone ridge, NY).

Determination of transfection efficiency. The Adv-1/GMCSF transfected and normal myeloma FO cells were harvested and fixed by $2 \%(\mathrm{w} / \mathrm{v})$ paraformaldehyde (PFA) for $1 \mathrm{~h}$. The cell membrane was permeated by PBS supplemented with
$0.5 \%(\mathrm{v} / \mathrm{v})$ Triton $\mathrm{X}-100$ and $0.5 \%(\mathrm{w} / \mathrm{v}) \mathrm{BSA}$ at room temperature for $15 \mathrm{~min}$. Then the cells were incubated with fluorescence (FITC)-conjugated anti-GM-CSF antibody $\left(0.5 \mu \mathrm{g} / 10^{6}\right.$ cells $)$ for $1 \mathrm{~h}$. The fluorescence was observed using a fluorescence microscope (Nikon, Japan). Normal myeloma FO cells were used as control.

Sandwich ELISA. The supernatant of Adv-1/GM-CSF transfected myeloma FO cells was collected. The amount of released GM-CSF in the supernatant was quantified by a sandwich ELISA kit (BD pharmingen, CA).

Immunization of mice with lyophilized tumor cells. Lyophilized transfected and normal myeloma FO cells $\left(1 \times 10^{6}\right)$ were dissolved with $100 \mu \mathrm{l}$ PBS, and mixed with equal volumes of complete Freund's adjuvant (CFA). Female BALB/c mice were injected subcutaneously in the flank with a $200 \mu 1$ mixture biweekly. One week after the second immunization, all of the mice were challenged with $1 \times 10^{6}$ myeloma FO cells. Then the mice were monitored for 55 days until all of the mice in the control group had died. Tumor sizes of tumorbearing mice were measured by a vernier caliper. The tumor volume was determined by the following equation: tumor volume $\left(\mathrm{mm}^{3}\right)=$ length $\mathrm{x}$ width $2 / 2$.

Flow cytometry assay. Myeloma FO cells were washed by PBS and incubated by 1:100 diluted sera from the immunized and control mice. The cells were then incubated with PEconjugated goat anti-mouse antibody (BD Pharmingen). Tcells isolated from mice splenocytes were incubated with PE-conjugated anti-CD3 antibody (BD Pharmingen) to confirm the purity. All samples were analyzed with a FACS caliber (Becton Dickinson, CA).

The CTL assay. The T-cells harvested from mouse spleen by nylon wool absorption were plated into 24-well plates containing $50 \mu \mathrm{g} / \mathrm{ml}$ mitomycin C (Union Pharmaceuticals, Beijing, P.R. China) pre-treated FO for $72 \mathrm{~h}$. The ratio of T-cells to FO cells was 5:1. T-cells were then harvested and cocultured with the target FO cells for $6 \mathrm{~h}$ in a 96-well Ubottom plate at various ratios. The supernatant was measured for lactate dehydrogenase (LDH) released from lysed cells using the CytoTox 96 cytotoxicity assay kit (Promega). Percentage of specific release of LDH was determined by the following equation: percent specific release $(\%)=($ experimental release - spontaneous T-cell release - spontaneous FO cell release) / (maximal FO cell release -spontaneous FO cell release) $\mathrm{x} 100$.

Statistical methods. Statistical significance was determined using Student's t-test.

\section{Results}

Transfection of myeloma FO cells. Myeloma FO cells were transfected by adenovirus containing recombinant GM-CSF (Adv-1/GM-CSF). The transfection efficiency was assayed by immunocytochemical staining with FITC-conjugated antiGM-CSF antibody. Compared with normal myeloma FO cells under both light and fluorescence microscopy, more than 


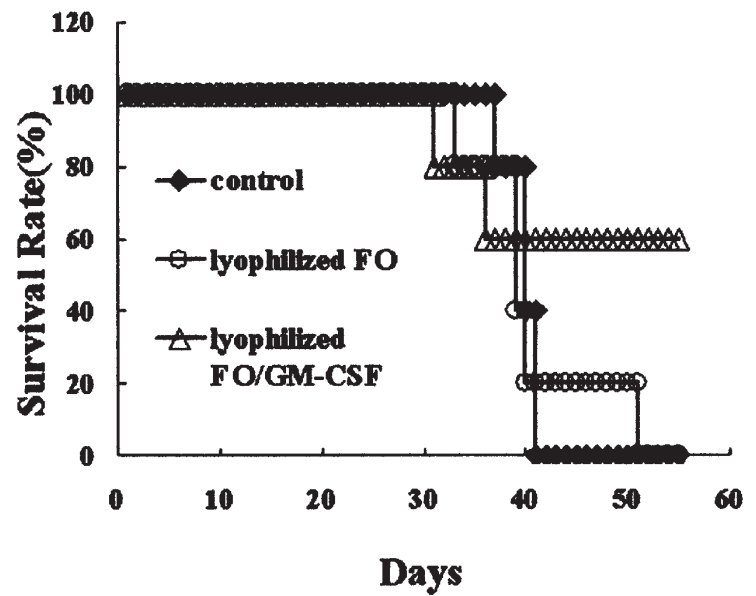

Figure 2. Anti-tumor immunity induced by lyophilized myeloma FO cells secreting GM-CSF. BALB/c mice were subcutaneously immunized twice with PBS (๑), lyophilized myeloma FO cells without transfection $(\circ)$ and lyophilized myeloma FO cells secreting GM-CSF $(\triangle)$, respectively. Mice were challenged with $1 \times 10^{6}$ myeloma FO cells one week after the last immunization. The survival rate was monitored for 55 days until all control mice had died. Every group comprised 5 mice.

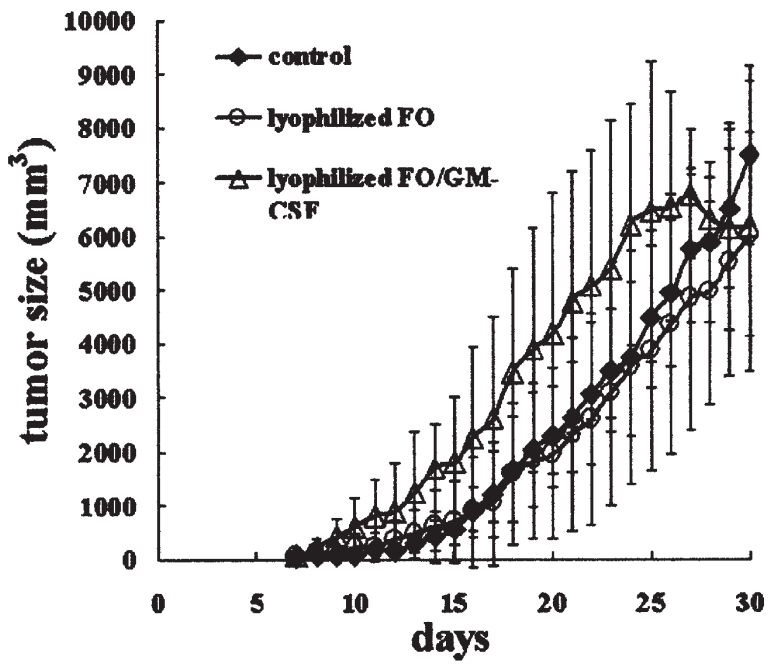

Figure 3. Tumor sizes of tumor-bearing mice in immune group and control group. BALB/c mice were subcutaneously immunized twice with PBS (•), lyophilized myeloma FO cells without transfection $(0)$ and lyophilized myeloma FO cells secreting GM-CSF $\triangle$ ), respectively. Mice were challenged with $1 \times 10^{6}$ myeloma FO cells one week after the last immunization. Tumor sizes of tumor-bearing mice were measured by a vernier caliper every day until mice started to die. The tumor volume was determined by the following equation: tumor volume $\left(\mathrm{mm}^{3}\right)=$ length $\mathrm{x}$ width ${ }^{2} / 2$.

$90 \%$ of transfected FO cells were positive for GM-CSF secretion (Fig. 1). Additionally, recombinant GM-CSF released in the supernatant from transfected cells was $240 \mathrm{ng} / 24 \mathrm{~h}$ per $10^{6}$ cells, as quantified by a sandwich ELISA.

Anti-tumor immunity induced by lyophilized myeloma cells. A tumor vaccination model was used to evaluate the induced anti-tumor immunity. Successful immune responses should help mice survive from the challenge of tumor cells. Female $\mathrm{BALB} / \mathrm{c}$ mice were immunized biweekly with PBS, lyophilized

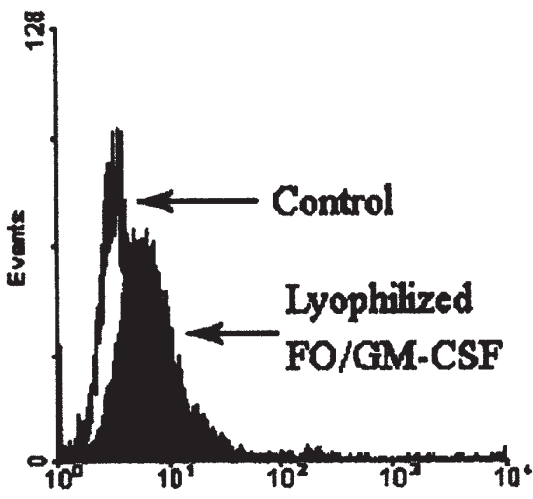

Figure 4. Induction of antibody against myeloma FO cells. Myeloma FO cells were incubated with 1:100 diluted serum of control $(\square)$ and immunized mice ( $\boldsymbol{\square})$, followed by PE-conjugated goat anti-mouse antibody. The immunocytochemical staining was analyzed by FACS.

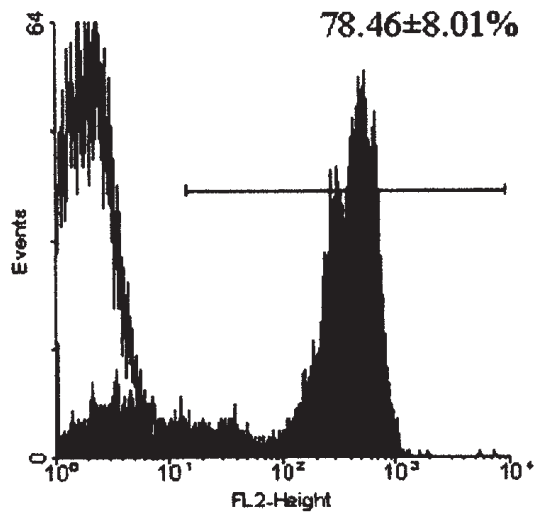

Figure 5. Purity of T-cells isolated from mice splenocytes. T-cells were enriched by nylon wool fiber column. The purity was analyzed by PEconjugated anti-CD3 antibody. The results were expressed as the mean \pm SD of 3 replicates.

myeloma FO and lyophilized myeloma FO secreting GM$\mathrm{CSF}$, respectively. One week after the second immunization, mice were subcutaneously injected with $1 \times 10^{6}$ myeloma FO cells. All control mice died within 40 days after the inoculation of FO cells. All mice immunized with lyophilized myeloma FO cells died within 51 days later. However, in the group of mice immunized with lyophilized myeloma FO cells secreting GM-CSF, $60 \%$ of mice survived without development of tumors (Fig. 2). At the same time, tumor sizes of the tumorbearing mice in the immunized group were not obviously different from those of the control mice (Fig. 3).

The dominant role of humoral immunity. We further investigated why lyophilized myeloma FO secreting GM-CSF induced strong anti-tumor immunity. Tumor-specific antibody response and cytotoxic $\mathrm{T}$-cells against $\mathrm{FO}$ cells from immunized mice were assayed by FACS and CTL assay, respectively. Myeloma FO cells were incubated with diluted serum from immunized and control mice. Immune serum from mice immunized with lyophilized FO myeloma cells secreting GM-CSF demonstrated specific recognition to FO cells in comparison with the control serum (Fig. 4), suggesting that a successful humoral immune response against 


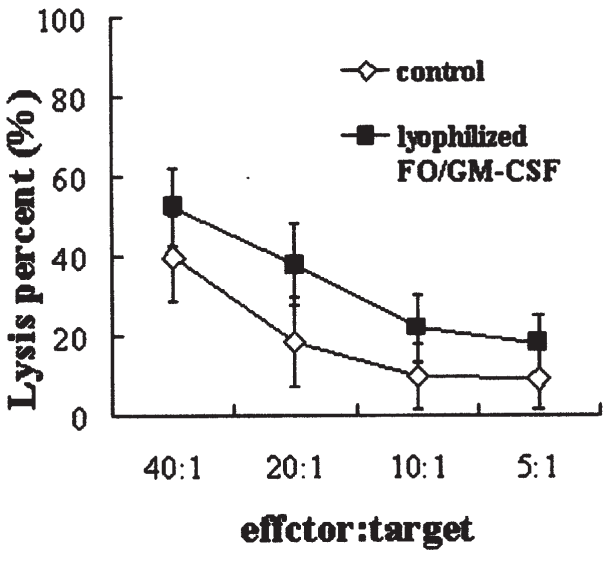

Figure 6. Function of cytotoxic T lymphocytes (CTLs) from immunized mice. After activation in vitro, CTLs from mice immunized with PBS (घ) and lyophilized myeloma FO cells secreting GM-CSF $(\diamond)$ were incubated with target myeloma cells at indicated ratios. $\mathrm{LDH}$ released from lysed FO myeloma cells was measured. The data were expressed as the mean $\pm \mathrm{SD}$ of 3 replicates.

myeloma FO cells was induced. Additionally, the lysis activity against FO cells of cytotoxic T lymphocytes was tested. T lymphocytes were isolated from mice splenocytes by a nylon wool fiber column. The purity of collected cells reached $78.46 \pm 8.01 \%$ (Fig. 5). In the CTL assay, the percentage of lysis increased along with the ratios of effector cells versus target cells. However, cytotoxicity mediated by T-cells from myeloma FO cells secreting GM-CSF immunized mice and the control mice was not dramatically different (Fig. 6). These data suggested that strong humoral immunity was raised in the present method, whereas the role of cellular immunity remained to be elucidated.

\section{Discussion}

In our previous study, a low dose of live myeloma FO cells induced a potent cellular immunity against tumor without foreign modulating factors, which inhibited the tumor growth in immunized BALB/c mice (17). In the present study, we investigated whether lyophilized myeloma FO cells could also induce anti-tumor immunity alone. In the myeloma vaccination model, lyophilized myeloma FO cells did not induce a strong immunity against tumor. However, the immunity could be greatly enhanced by transfection of myeloma FO cells with Adv-1/GM-CSF, which prevented $60 \%$ of $\mathrm{BALB} / \mathrm{c}$ mice from developing tumors.

The mechanism of the anti-tumor immunity was also analyzed. Higher levels of antibodies in the immune serum against myeloma FO cells were detected using FACS, suggesting the humoral immune response against tumor cells was induced. In the CTL assay, there was an obvious but not dramatic difference between T-cells from myeloma FO cell expressing GM-CSF immunized mice and the control mice. These results suggested that cytotoxic $\mathrm{T}$ lymphocytes were also activated. In conclusion, humoral immunity played an important role in the protective immunity. It was different from the immunity induced by live cells or irradiated tumor cell secreting cytokines. This may be due to the different types of the vaccines. Cell-type vaccines could provide three signals, including antigens presented in MHC molecules, costimulatory factors and cytokines (18). Additionally, they are arrayed in a certain dimensional form on cell membrane, which could be recognized by pattern recognition receptors (PRR) on lymphocytes (19). Cellular immunity was then activated. However, lyophilized tumor cells were mainly in the protein types, which were lacking in costimulatory factors and special dimensional arrays. Therefore, the stronger humoral immunity was usually induced by protein vaccines, but not the cellular immunity. Only if the PRR were activated, such as pulsed dendritic cells with tumor antigens, could cellular immunity be induced (12).

Traditionally, tumor cell lysates were prepared by sonication or freeze and thaw (20). Protein antigens were likely to be proteolysed under these conditions. In the present method, tumor lysates were prepared by lyophilization, which prevented the possibility of proteolysis. Compared with our previous method, the present method was safer than immunization with live tumor cells and it is promising for use when developing safe and potent tumor vaccines for clinical myeloma immunotherapy.

\section{Acknowledgements}

This study was supported in part by the Ministry of Education of P.R. China (20020284025 and IRT0430) and by Nanjing University (985-I-FZS).

\section{References}

1. Shimizu K, Goldfarb M, Suard Y, et al: Three human transforming genes are related to the viral ras oncogenes. Proc Natl Acad Sci USA 80: 2112-2116, 1980.

2. Hanahan D and Weinberg RA: The hallmark of cancer. Cell 100: $57-70,2000$

3. Kawakami Y, Eliyahu S, Sakaguchi K, et al: Identification of the immunodominant peptides of the MART-1 human melanoma antigen recognized by the majority of HLA-A2 restricted tumor infiltrating lymphocytes. J Exp Med 180: 347-352, 1994.

4. Boel P, Wildmann C, Sensi ML, et al: BAGE: a new gene encoding an antigen recognized on human melanomas by 22 cytolytic T lymphocytes. Immunity 2: 167-175, 1995.

5. Kawakami Y, Eliyahu S, Jennings C, et al: Recognition of multiple epitopes in the human melanoma antigen gp100 by tumor infiltrating T-lymphocytes associated with in vivo tumor regression. J Immunol 154: 3961-3968, 1995.

6. Parkhurst MR, Fitzgerald EB, Southwood S, Sette A, Rosenberg SA and Kawakami Y: Identification of a shared HLA-A*0201-restricted T-cell epitope from the melanoma antigen tyrosinase-related protein 2 (TRP2). Cancer Res 58: 4895-4901, 1998.

7. Adams GP and Weiner LM: Monoclonal antibody therapy of cancer. Nat Biotechnol 23: 1147-1157, 2005.

8. Sensi M, Traversari C, Radrizzani M, et al: Cytotoxic Tlymphocyte clones from different patients display limited Tcell-receptor variable-region gene usage in HLA-A2-restricted recognition of the melanoma antigen Melan-A/MART-1. Proc Natl Acad Sci USA 92: 5674-5678, 1995.

9. Weiner LM: An overview of monoclonal antibody therapy of cancer. Semin Oncol 26 (4 suppl 12): 41-50, 1999

10. Vogel CL, Cobleigh MA, Tripathy D, et al: Efficacy and safety of trastuzumab as a single agent in first-line treatment of HER2overexpressing metastatic breast cancer. J Clin Oncol 20: 719-726, 2002.

11. Mach N and Dranoff G: Cytokine-secreting tumor cell vaccines. Curr Opin Immunol 12: 571-575, 2000.

12. Mayordomo JI, Zorina T, Storkus WJ, et al: Bone marrowderived dendritic cells pulsed with synthetic tumor peptides elicit protective and therapeutic antitumor immunity. Nat Med 1: $1297-1302,1995$ 
13. Gong J, Chen D and Kufe D: Induction of antitumor activity by immunization with fusions of dendritic and carcinoma cells. Nat Med 3: 558-561, 1997.

14. Lotze MT, Chang AE, Seipp CA, Simpson C, Vetto JT and Rosenberg SA: High-dose recombinant interleukin-2 in the treatment of patients with disseminated cancer. JAMA 256: 3117-3124, 1986.

15. Soiffer RJ, Robertson MJ, Murray C, Cochran K and Ritz J: Interleukin-12 augments cytolytic activity of peripheral blood lymphocytes from patients with hematologic and solid malignancies. Blood 82: 2790-2796, 1993.

16. Soiffer R, Hodi FS, Haluska F, et al: Vaccination with irradiated, autologous melanoma cells engineered to secrete granulocytemacrophage colony-stimulating factor by adenoviral-mediated gene transfer augments antitumor immunity in patients with metastatic melanoma. J Clin Oncol 21: 3343-3350, 2003.
17. Wu S, Wang S-Q, Zhang J, Tan X-Y and Liu J-N: Native antitumor responses elicited by immunization with a low dose of unmodified live tumor cells. Int J Oncol 28: 731-736, 2006.

18. Curtsinger JM, Schmidt CS, Mondino A, Lins DC, Kedl RM, Jenkins MK and Mescher MF: Inflammatory cytokines provide a third signal for activation of naive CD41 and CD81 T cells. J Immunol 162: 3256-3262, 1999.

19. Allavena P, Chieppa M, Monti P and Piemonti L: From pattern recognition receptor to regulator of homeostasis: the doublefaced macrophage mannose receptor. Crit Rev Immunol 24: 179-192, 2004.

20. Herr W, Ranieri E, Olson W, Zarour H, Gesualdo L and Storkus WJ: Mature dendritic cells pulsed with freeze-thaw cell lysates define an effective in vitro vaccine designed to elicit EBV-specific CD4+ and CD8+ T lymphocyte responses. Blood 96: $1857-1864,2000$. 